\title{
Langevin models for shear-stress fluctuations in flows of viscoelastic liquids
}

\author{
Jeppe C. Dyre \\ Institut for Studiet af Matematik og Fysik samt deres Funktioner i Undervisning, Forskning og Anvendelser, \\ Roskilde Universitetscenter, Postbox 260, DK-4000 Roskilde, Denmark
}

(Received 1 March 1993)

\begin{abstract}
From the principle of virtual work it is shown that, if equilibrium stress fluctuations are described by a Langevin equation, there is only one possible extension of this equation to describe stress fluctuations in a shear flow. It is shown that the resulting equation is consistent with linear-response theory. The formalism developed may be looked upon as a method for extending differential constitutive relations to incorporate thermal fluctuations. A few simple models are discussed as illustrations. These include a model where the stress fluctuates freely below a certain limit, and a model constructed to mimic the Tobolsky-Eyring phenomenological theory [J. Chem. Phys. 11, 125 (1942)] of viscoelastic liquids. It is concluded that these and similar models, however, do not realistically describe real polymeric liquids. To reach this goal, models involving several stress degrees of freedom will have to be considered.
\end{abstract}

PACS number(s): 82.70. $-\mathrm{y}, 05.40 .+\mathrm{j}, 03.40 . \mathrm{Gc}$

\section{INTRODUCTION}

Presently, the most popular models of the viscoelasticity of polymeric liquids are based on a microscopic description, where the bead coordinates are the basic degrees of freedom [1,2]. These models are rather successful but, unfortunately, also quite complicated to solve. The present paper investigates the more phenomenological approach to take as a basic degree of freedom the quantity of main interest, the shear stress. In part, this represents a return to ideas proposed by Eyring, Tobolsky, Andrews, and Hofman-Bang many years ago [3-5]. Here, however, a formalism is developed which is consistent with statistical mechanics. This is done by assuming a Langevin equation for the shear-stress dynamics in equilibrium. From this the stress fluctuations may be calculated and thereby, via the fluctuation-dissipation theorem, the linear frequency-dependent viscosity may be calculated. The main result of the paper is a proof that the nonlinear response to any shear displacement is uniquely determined by the requirement that the principle of virtual work is obeyed. It is shown that this principle ensures that linear-response theory is obeyed, a sine qua non requirement (Sec. II). In Sec. III a few simple models are worked out. Finally, in Sec. IV a discussion is given. It is concluded that, in order to model realistically reality, the proposed formalism must be generalized to include spatially varying stresses.

\section{UNIQUE COUPLING OF DEFORMATION TO STRESS FLUCTUATIONS}

Here and henceforth the term "stress" refers to the shear stress $\sigma_{x y}$, while the corresponding $x-y$ shear rate is denoted by $\dot{\gamma}(t)$. The fluctuation-dissipation theorem [2] allows a calculation of the frequency-dependent viscosity $\eta(\omega)$ in terms of equilibrium stress fluctuations. The theorem states that the stress relaxation modulus $G(t)$, which is characterized by

$$
\eta(\omega)=\int_{0}^{\infty} G(t) e^{-i \omega t} d t
$$

is given by

$$
G(t)=\beta \frac{\langle s(0) s(t)\rangle_{0}}{V} .
$$

Here, a sample of volume $V$ is considered, $s$ is the "total" stress (i.e., $\sigma_{x y}=s / V$, and $\beta=1 / k_{B} T$. The subscript zero on the right-hand side of Eq. (2) is introduced to remind one that the autocorrelation function refers to fluctuations in thermal equilibrium. If $\mathbf{R}_{i}$ is the coordinate vector of the $i$ th bead and $\mathbf{F}_{i}$ is the force on the $i$ th bead, the quantity $s$ is given [2] by

$$
s=-\sum_{i} R_{i, x} F_{i, y} .
$$

[Note that the relaxation modulus of Eq. (2) has a welldefined limit for $V \rightarrow \infty$ : this is the usual macroscopic shear-stress relaxation modulus.]

From now on the following simple model is adopted. The liquid is regarded as divided into regions whose stresses fluctuate independently. A discussion of this rather severe approximation is postponed to Sec. IV. There is just one relevant degree of freedom in the model, the quantity $s$ of Eq. (3), where the sum is now restricted to one region. The quantity $s$ has dimension energy, but will still be referred to as the "stress." It is convenient also to redefine "viscosity" by absorbing the region volume, so that viscosity is from now on simply $\langle s\rangle / \dot{\gamma}$. With these definitions Eqs. (1) and (2) become

$$
\eta(\omega)=\beta \int_{0}^{\infty}\langle s(0) s(t)\rangle_{0} e^{-i \omega t} d t
$$

We remind the reader that Eq. (4) is equivalent to saying that the average stress for small shear rate $\dot{\gamma}(t)$ is given by

$$
\langle s(t)\rangle_{\dot{\gamma}}=\beta \int_{0}^{\infty}\langle s(0) s(\tau)\rangle_{0} \dot{\gamma}(t-\tau) d \tau .
$$

According to statistical mechanics, the probability of an $s$ 
fluctuation in equilibrium is given by the free energy as a function of $s, F(s)$. If $\Gamma$ denotes the complete set of microscopic coordinates referring to one region, and $s(\Gamma)$ and $E(\Gamma)$ denote, respectively, the value of $s$ and of energy in state $\Gamma, F(s)$ is given by

$$
e^{-\beta F(s)}=\int e^{-\beta E(\Gamma)} \delta(s-s(\Gamma)) d \Gamma .
$$

It is assumed that $F(s) \rightarrow \infty$ as $|s| \rightarrow \infty$, and that $F(s)=F(-s)$. Note that the free energy of one region is given by

$$
e^{-\beta F}=\int_{-\infty}^{\infty} e^{-\beta F(s)} d s
$$

Now the further assumption is made that the $s$ fluctuations in equilibrium are described by a Langevin equation,

$$
\dot{s}=-\mu \frac{d F}{d s}+\xi(t)
$$

Here, $\mu$ is the "mobility" ("velocity"/"force") which determines the time scale, and $\xi(t)$ is a Gaussian whitenoise term:

$$
\left\langle\xi(t) \xi\left(t^{\prime}\right)\right\rangle=2 \mu k_{B} T \delta\left(t-t^{\prime}\right) .
$$

While Eq. (8) is a completely phenomenological postulate, it has the crucial property $[2,6,7]$ that the stationary $s$-probability distribution $P_{0}(s)$ is that required by statistical mechanics:

$$
P_{0}(s)=N^{-1} e^{-\beta F(s)} .
$$

Any initial probability distribution converges to $P_{0}(s)$ as $t \rightarrow \infty$; the equation governing this is the well-known Smoluchowski equation $[2,6,7]$ (sometimes referred to as the Fokker-Planck equation or just the diffusion equation)

$$
\frac{\partial P}{\partial t}=\frac{\partial}{\partial s}\left[\mu \frac{d F}{d s} P+\mu k_{B} T \frac{\partial P}{\partial s}\right) .
$$

A substitution of Eq. (10) into Eq. (11) confirms that $P_{0}(s)$ is the stationary solution.

How is the $s$ dynamics changed when the liquid flows? The simplest way to modify Eq. (8) is to add an extra term coupling to $\dot{\gamma}(t)$, writing

$$
\dot{s}=-\mu \frac{d F}{d s}+\dot{\gamma}(t) J(s)+\xi(t) .
$$

In Eq. (12) the shear rate plays the role of an external field. Obviously, $J(s)$ is a kind of stress-dependent infinite frequency shear modulus. The Smoluchowski equation corresponding to Eq. (12) is

$$
\frac{\partial P}{\partial t}=\frac{\partial}{\partial s}\left[\left[\mu \frac{d F}{d s}-\dot{\gamma}(t) J\right] P\right]+\mu k_{B} T \frac{\partial^{2} P}{\partial s^{2}} .
$$

We now proceed to show first that $J(s)$ is uniquely determined by the principle of virtual work. Then it is shown that, with this choice of $J(s)$, linear-response theory [Eqs. (1) and (2)] is reproduced in the small shear rate limit, as is necessary to have a consistent theory.

For any probability distribution $P(s)$ the dynamical free energy $A$ is defined [2] by

$$
A=\int_{-\infty}^{\infty} d s P(s)\left\{F(s)+k_{B} T \ln [P(s)]\right\} .
$$

The principle of virtual work $[2,8]$ says that after the virtual displacement

$$
\dot{\gamma}(t)=\delta \gamma \delta(t),
$$

$A$ is changed by

$$
\delta A=\langle s\rangle \delta \gamma \text {. }
$$

From Eq. (14) the variation in $A$ is given by

$$
\delta A=\int_{-\infty}^{\infty} d s \delta P(s)\left\{F(s)+k_{B} T \ln [P(s)]\right\} .
$$

Substituting Eq. (15) into Eq. (13) and integrating over a small interval around 0 gives, to lowest order in $\delta \gamma$,

$$
\delta P=-\delta \gamma \frac{\partial}{\partial s}(J P)
$$

By combining Eqs. (17) and (18) one finds by partial integrations

$$
\begin{aligned}
\delta A & =-\delta \gamma \int_{-\infty}^{\infty} d s\left[F \frac{\partial}{\partial s}(J P)+k_{B} T \ln (P) \frac{\partial}{\partial s}(J P)\right] \\
& =\delta \gamma \int_{-\infty}^{\infty} d s\left[\frac{d F}{d s} J P+k_{B} T \frac{\partial P}{\partial s} J\right] \\
& =\delta \gamma \int_{-\infty}^{\infty} d s\left[\frac{d F}{d s} J-k_{B} T \frac{d J}{d s}\right] P .
\end{aligned}
$$

If this is to be consistent with Eq. (16) for all $P(s), J(s)$ must obey

$$
\frac{d J}{d s}=\beta \frac{d F}{d s} J-\beta s .
$$

The solution of this equation is

$$
J(s)=e^{\beta F(s)} \int_{s}^{\infty} d s^{\prime} \beta s^{\prime} e^{-\beta F\left(s^{\prime}\right)} .
$$

All other solutions lead to an exponentially increasing $J(s)$ and thereby an inconsistent model where $s$ runs off to infinity in any shear flow. For $\beta \rightarrow \infty$ Eq. (21) implies $J(s)=s /(d F / d s)$.

Next it is shown that the $J(s)$ of Eq. (21) ensures that linear-response theory is reproduced. First Eq. (12) is rewritten as

$$
\dot{s}=-\mu \frac{\partial}{\partial s}[F-\dot{\gamma}(t) \Phi]+\xi(t)
$$

where

$$
\frac{d \Phi}{d s}=\frac{J}{\mu} .
$$

Equation (22) shows that the coupling to the shear displacement "field" appears as an extra term $-\dot{\gamma}(t) \Phi(s)$ in the Hamiltonian. In the small-shear-rate limit, linearresponse theory applied to Eq. (22) leads [2] to

$$
\langle s(t)\rangle_{\dot{\gamma}}=\beta \int_{0}^{\infty} \dot{\gamma}(t-\tau) \frac{-d}{d \tau}\langle\Phi(\tau=0) s(\tau)\rangle_{0} d \tau .
$$


Equation (24) is consistent with Eq. (5) if

$$
-\frac{d}{d \tau}\langle\Phi(\tau=0) s(\tau)\rangle_{0}=\langle s(0) s(\tau)\rangle_{0} .
$$

To prove Eq. (25) note that the $\Phi-s$ correlation function is given by

$$
\begin{aligned}
& \langle\Phi(\tau=0) s(\tau)\rangle_{0} \\
& \quad=\int_{-\infty}^{\infty} d s^{\prime} s^{\prime} \int_{-\infty}^{\infty} d s P_{0}(s) \Phi(s) G_{0}\left(s, s^{\prime} ; \tau\right),
\end{aligned}
$$

where $G_{0}\left(s, s^{\prime} ; \tau\right)$ is the equilibrium Green's function, i.e., the probability of finding stress $s^{\prime}$ at time $t$ if the stress were $s$ at $t=0$. By substituting into this expression the time-integrated version of detailed balance $P_{0}(s) G_{0}\left(s, s^{\prime} ; \tau\right)=P_{0}\left(s^{\prime}\right) G_{0}\left(s^{\prime}, s ; \tau\right)$, one finds

$$
\begin{aligned}
& -\frac{d}{d \tau}\langle\Phi(\tau=0) s(\tau)\rangle_{0} \\
& \quad=-\int_{-\infty}^{\infty} d s^{\prime} s^{\prime} P_{0}\left(s^{\prime}\right) \int_{-\infty}^{\infty} d s \Phi(s) \frac{\partial G_{0}}{\partial \tau}\left(s^{\prime}, s ; \tau\right) .
\end{aligned}
$$

The Green's function considered as a function of the second variable satisfies Eq. (11), of course, and therefore one gets

$$
\begin{aligned}
-\frac{d}{d \tau}\langle\Phi(\tau=0) s(\tau)\rangle_{0} & =-\int_{-\infty}^{\infty} d s^{\prime} s^{\prime} P_{0}\left(s^{\prime}\right) \int_{-\infty}^{\infty} d s \Phi(s)\left[\mu \frac{\partial}{\partial s}\left[\frac{d F}{d s} G_{0}\right]+\frac{\mu}{\beta} \frac{\partial^{2} G_{0}}{\partial s^{2}}\right] \\
& =\int_{-\infty}^{\infty} d s^{\prime} s^{\prime} P_{0}\left(s^{\prime}\right) \int_{-\infty}^{\infty} d s\left[J(s) \frac{d F}{d s}-\frac{1}{\beta} \frac{d J}{d s}\right] G_{0}\left(s^{\prime}, s ; \tau\right) .
\end{aligned}
$$

Because $J(s)$ satisfies Eq. (20) it is now clear that Eq. (25) is obeyed.

\section{SOME SIMPLE MODELS}

\section{A. Gaussian model}

In this model the free energy is assumed to be quadratic in $s$ :

$$
F(s)=\frac{1}{2} \alpha s^{2} .
$$

The equilibrium $s$-probability distribution is a Gaussian

$$
P_{0}(s)=\left(\frac{\alpha \beta}{2 \pi}\right)^{1 / 2} e^{-(1 / 2) \alpha \beta s^{2}},
$$

which implies

$$
\left\langle s^{2}\right\rangle_{0}=\frac{1}{\alpha \beta} \text {. }
$$

The "equation of motion" for $s$ is

$$
\dot{s}(t)=-\mu \alpha s(t)+\xi(t) .
$$

If Eq. (32) is multiplied by $s(0)$ and averaged, one finds

$$
\frac{d}{d t}\langle s(0) s(t)\rangle_{0}=-\mu \alpha\langle s(0) s(t)\rangle_{0} \quad(t>0) .
$$

The solution of Eq. (33) which satisfies Eq. (31) is

$$
\langle s(0) s(t)\rangle_{0}=\frac{1}{\alpha \beta} e^{-\mu \alpha t} \quad(t>0) .
$$

The calculation of $J(s)$ is straightforward; from Eq. (21) one finds

$$
J(s)=e^{(1 / 2) \alpha \beta s^{2}} \int_{s}^{\infty} \beta s^{\prime} e^{-(1 / 2) \alpha \beta s^{\prime 2}} d s^{\prime}=\frac{1}{\alpha} .
$$

The Smoluchowski equation (13) thus is

$$
\frac{\partial P}{\partial t}=\frac{\partial}{\partial s}\left[\left[\mu \alpha s-\frac{\dot{\gamma}(t)}{\alpha}\right] P\right]+\mu k_{B} T \frac{\partial^{2} P}{\partial s^{2}} \text {. }
$$

From Eq. (36) a simple equation for the average $\langle s(t)\rangle_{\dot{\gamma}}$ may be derived by application of the obvious identity

$$
\frac{d}{d t}\langle s(t)\rangle_{\dot{\gamma}}=\int_{-\infty}^{\infty} s \frac{\partial P}{\partial t}(s, t) d s .
$$

Substituting Eq. (36) into Eq. (37), one finds after partial integrations

$$
\frac{d}{d t}\langle s(t)\rangle_{\dot{\gamma}}=-\mu \alpha\langle s(t)\rangle_{\dot{\gamma}}+\frac{\dot{\gamma}(t)}{\alpha} .
$$

As usual it is assumed that the shear rate "field" $\dot{\gamma}(t)$ is introduced gradually in the distant past. The solution of Eq. (38) which vanishes as $t \rightarrow-\infty$ is

$$
\langle s(t)\rangle_{\dot{\gamma}}=\int_{-\infty}^{t} \frac{\dot{\gamma}\left(t^{\prime}\right)}{\alpha} e^{-\mu \alpha\left(t-t^{\prime}\right)} d t^{\prime} .
$$

By means of Eq. (34) this may be rewritten as

$$
\langle s(t)\rangle_{\dot{\gamma}}=\beta \int_{0}^{\infty}\langle s(0) s(\tau)\rangle_{0} \dot{\gamma}(t-\tau) d \tau .
$$

Equation (40) is nothing but the prediction of linearresponse theory [Eq. (5)]. Thus, the Gaussian model is linear for all displacements.

\section{B. Box model}

This model is defined by

$$
F(s)=\left\{\begin{array}{ll}
0, & |s|<s_{0} \\
\infty, & |s|>s_{0}
\end{array} .\right.
$$

The model is named after the box model in elementary quantum mechanics; it should not be confused with the box-model distribution of relaxation times sometimes used in rheology [9]. Since there is a maximum value of the stress, the box model must exhibit shear thinning at large shear rates. To find the nonlinear viscosity $\eta(\dot{\gamma})$ we need to determine $J(s)$ first. In the present case Eq. (20) reduces to $d J / d s=-\beta s$. The solution of this equation, which satisfies the boundary conditions $J\left(-s_{0}\right)=J\left(s_{0}\right)=0$, is 


$$
J(s)=\frac{\beta}{2}\left(s_{0}^{2}-s^{2}\right) .
$$

The $\Phi$ function of Eq. (23) thus becomes

$$
\Phi(s)=\frac{\beta}{2 \mu}\left(s_{0}^{2} s-\frac{1}{3} s^{3}\right) .
$$

For a given shear rate $\dot{\gamma}$, the stationary solution of Eq. (13) is

$$
P(s)=N^{-1} e^{\beta \dot{\gamma} \Phi(s)}, \quad\left(|s|<s_{0}\right),
$$

where $N$ is a normalization constant, and $P(s)=0$ for $|s|>s_{0}$. If the dimensionless shear rate

$$
\dot{\gamma}^{*}=\frac{\beta^{2} s_{0}^{3}}{\mu} \dot{\gamma}
$$

is introduced, one finds from Eq. (44)

$$
\langle s\rangle_{\dot{\gamma}}=s_{0} \frac{\int_{-1}^{1} t e^{\dot{\gamma}^{*}\left[(t / 2)-\left(t^{3} / 6\right)\right]} d t}{\int_{-1}^{1} e^{\dot{\gamma}^{*}\left[(t / 2)-\left(t^{3} / 6\right)\right]} d t} .
$$

From this the nonlinear viscosity $\eta=\langle s\rangle / \dot{\gamma}$ is readily evaluated. The result is shown in Fig. 1(a) and is not unlike that seen in polymeric liquids. In the linear limit one finds, by expanding Eq. (46) to first order in $\dot{\gamma}^{*}$, the shear rate independent viscosity $\eta_{0}$ given by

$$
\eta_{0}=\frac{2 \beta^{2} s_{0}^{4}}{15 \mu} .
$$

The equilibrium dynamics is governed by the simple diffusion equation [Eq. (11)]

$$
\frac{\partial P}{\partial t}=\frac{\mu}{\beta} \frac{\partial^{2} P}{\partial s^{2}},
$$

subject to the boundary conditions

$$
\frac{\partial P}{\partial s}\left(s=-s_{0}\right)=\frac{\partial P}{\partial s}\left(s=s_{0}\right)=0 .
$$

The eigenfunctions of this problem are $\cos \left[n \pi\left(s_{0}-s\right) /\left(2 s_{0}\right)\right] \quad(n=0,1,2, \ldots)$. From this one finds by standard methods [10] that the equilibrium Green's function is given by

$$
\begin{aligned}
G_{0}\left(s, s^{\prime} ; t\right)=\frac{1}{2 s_{0}}+\sum_{n=1}^{\infty} & \frac{1}{s_{0}} e^{-\omega_{n} t} \cos \left[\lambda_{n}\left(s_{0}-s\right)\right] \\
& \times \cos \left[\lambda_{n}\left(s_{0}-s^{\prime}\right)\right],
\end{aligned}
$$

where

$$
\lambda_{n}=\frac{\pi}{2 s_{0}} n, \quad \omega_{n}=\frac{\mu}{\beta} \lambda_{n}^{2} \quad(n=1,2, \ldots) .
$$

It is now easy to derive an analytical expression for the frequency-dependent viscosity $\eta(\omega)$. Since the equilibrium probability distribution is $P_{0}(s)=1 /\left(2 s_{0}\right)$, the autocorrelation function becomes

$$
\langle s(0) s(t)\rangle_{0}=\int_{-s_{0}}^{s_{0}} \frac{d s}{2 s_{0}} \int_{-s_{0}}^{s_{0}} d s^{\prime} s s^{\prime} G_{0}\left(s, s^{\prime} ; t\right) .
$$

From Eqs. (50) and (52) and the fluctuation-dissipation theorem [Eq. (4)], one finds

$$
\eta(\omega)=\frac{32 \beta s_{0}^{2}}{\pi^{4}} \sum_{\text {odd } n} \frac{1}{n^{4}\left(i \omega+\omega_{n}\right)} .
$$

In the zero-frequency limit Eq. (53) reduces to Eq. (47) via the identity [11]

$$
\frac{1}{1^{6}}+\frac{1}{3^{6}}+\frac{1}{5^{6}}+\cdots=\frac{\pi^{6}}{960} \text {. }
$$

Figure 1(b) shows the real part of $\eta(\omega)$. The spectrum contains infinitely many relaxation times but these are hardly visible, being completely dominated by the fundamental frequency $\omega_{1}$. In effect, $\eta(\omega)$ is almost indistinguishable from the prediction of a simple Maxwell model where $\operatorname{Re} \eta(\omega)$ is proportional to $\omega^{-2}$ for $\omega \rightarrow \infty$.

Only in the relaxation towards equilibrium from a
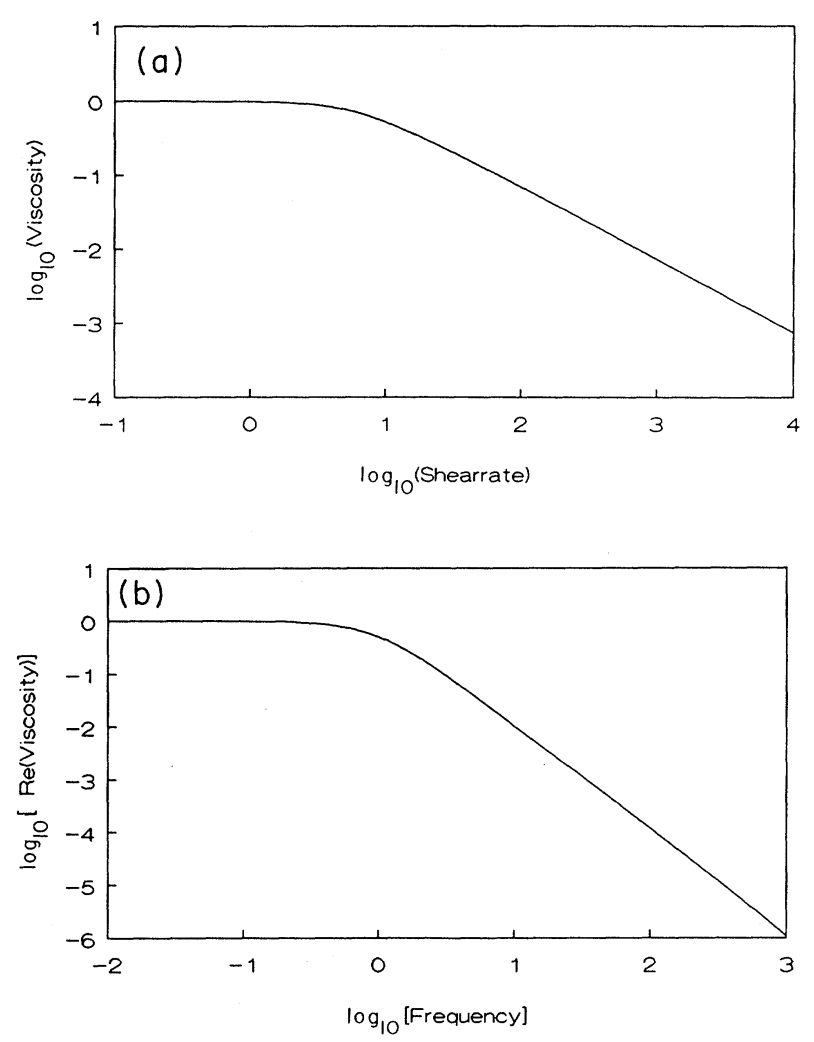

FIG. 1. Box-model predictions for the viscosity. (a) shows a log-log plot of the steady-state viscosity relative to the linear viscosity, as a function of the dimensionless shear rate given by Eq. (45). At large shear rates the viscosity varies as the inverse of the shear rate; this is a consequence of there being a maximum possible stress in the model. (b) shows a log-log plot of the real part of the frequency-dependent viscosity relative to the zero-frequency viscosity, as a function of the dimensionless frequency $\omega / \omega_{1}$. Despite the fact that the model has infinitely many relaxation times, the longest relaxation time dominates the frequency dependence completely. In effect, the frequency dependence is almost indistinguishable from that of a standard Maxwell model where the real part of the viscosity varies as $\omega^{-2}$ at large frequencies. 
strongly nonequilibrium state do the higher harmonics give significant contributions. If $\langle s(t)\rangle_{s}$ denotes the average stress, given the value $s$ at $t=0$, one has obviously

$$
\langle s(t)\rangle_{s}=\int_{-s_{0}}^{s_{0}} s^{\prime} G_{0}\left(s, s^{\prime} ; t\right) d s^{\prime} .
$$

Relaxation from a state with probability distribution $P(s)$ at $t=0$ is thus given by

$$
\langle s(t)\rangle_{P}=\int_{-s_{0}}^{s_{0}}\langle s(t)\rangle_{s^{\prime}} P\left(s^{\prime}\right) d s^{\prime} .
$$

Two well-known examples are stress relaxation after cessation of a steady flow, and stress relaxation after a sudden shear displacement starting from equilibrium [1]. In the first case, the probability distribution $P(s)$ is given by Eq. (44) at $t=0$. In the latter case, after the sudden shear displacement given by $\dot{\gamma}(t)=\gamma_{0} \delta(t)$, it is possible to show that $P(s)$ is given by

$$
P(s)=\frac{2 \lambda s_{0}}{\left[(1+\lambda) s_{0}-(1-\lambda) s\right]^{2}}, \quad \lambda=e^{-\beta \gamma_{0} s_{0}} .
$$

In both cases $P(s)$ is strongly peaked around $s=s_{0}$. From Eq. (50) we find

$$
\langle s(t)\rangle_{s}=\frac{2}{s_{0}} \sum_{\text {odd } n} \frac{1}{\lambda_{n}^{2}} e^{-\omega_{n} t} \cos \left[\lambda_{n}\left(s_{0}-s\right)\right] .
$$

This implies

$s-\langle s(t)\rangle_{s}=\frac{2}{s_{0}} \sum_{\text {odd } n} \frac{1}{\lambda_{n}^{2}}\left(1-e^{-\omega_{n} t}\right) \cos \left[\lambda_{n}\left(s_{0}-s\right)\right]$.

The interpretation of Eq. (59) is as follows. Whenever $t$ is so large that $\lambda_{n}\left(s_{0}-s\right)<1$ for all $n$ with $\omega_{n} t<1$, the cosine factor may be ignored altogether, and below a limiting $n=p$ given by $\omega_{p} t=1$, the exponential may be expanded to first order. Writing $p=a t^{-1 / 2}$, one has

$$
s-\langle s(t)\rangle_{s} \simeq C_{1} \int_{1}^{p} t \mathrm{dn}+C_{2} \int_{p}^{\infty} \frac{d n}{n^{2}} \propto t^{1 / 2},
$$

where $C_{1}$ and $C_{2}$ are constants. At very short times the quantity $s-\langle s(t)\rangle_{s}$ is exponentially close to zero. Then according to Eq. (60) comes a range of times where this quantity increases like $t^{1 / 2}$, and finally it converges to $s$. A similar result applies to relaxation from a state with probability distribution $P(s, t=0)$, since

$$
\langle s(0)\rangle_{P}-\langle s(t)\rangle_{P}=\int_{-s_{0}}^{s_{0}}\left[s-\langle s(t)\rangle_{s}\right] P(s, t=0) d s .
$$

If the width of $P(s, t=0), \Delta s$, is defined by the integral of $P$ from $s_{0}-\Delta s$ to $s_{0}+\Delta s$ being $\frac{1}{2}$, it is not hard to show that

$$
\langle s(0)\rangle_{P}-\langle s(t)\rangle_{P}\left\{\begin{array}{l}
\simeq 0, \quad t<t_{1} \\
\propto t^{1 / 2}, \quad t_{1}<t<t<t_{2} \\
\simeq\langle s(0)\rangle, \quad t_{2}<<t
\end{array},\right.
$$

where

$$
t_{1}=\frac{\beta}{\mu}(\Delta s)^{2}, \quad t_{2}=\frac{\beta}{\mu} s_{0}^{2} .
$$

In the case of relaxation after a sudden cessation of a steady shear flow, $\Delta s$ is given by [compare Eq. (44)]

$$
(\Delta s)^{2} \simeq \frac{\mu}{s_{0} \beta^{2}} \frac{1}{\dot{\gamma}} .
$$

In the case of a sudden large shear displacement, $\gamma_{0}, \Delta s$ is given by [compare Eq. (57)]

$$
\Delta s \simeq 2 s_{0} e^{-\beta \gamma_{0} s_{0}} .
$$

Note that, in both cases, the non-Debye character of the relaxation is apparent only because we have considered the quantity $\langle s(0)\rangle_{P}-\langle s(t)\rangle_{P}$. If one looks at just $\langle s(t)\rangle_{P}$, this quantity would be hard to distinguish from a simple exponential decay in time.

\section{Cosine hyperbolic model}

A phenomenological model for stress relaxation was proposed by Tobolsky and co-workers in the 1940s [3-5]. The model is a Maxwell element consisting of a Hooke's law spring and a non-Newtonian dashpot in series, the viscosity of the dashpot obeying the Eyring viscosity equation. The model leads to

$$
\dot{\gamma}=A \dot{s}+B \sinh \left(\frac{s}{s_{0}}\right),
$$

where $A, B$, and $s_{0}$ are constants. Equation (66) reproduces Eyring's viscosity equation and predicts a logarithmic stress relaxation at constant extension: At large $s(t=0)$ one has approximately $\dot{s}=-\operatorname{const} \times \exp \left(s / s_{0}\right)$, which implies at intermediate times [4]

$$
s(t) \cong \alpha-\beta \ln (t) .
$$

Both predictions of Eq. (66) mimic experiment on typical polymeric liquids. The model, however, does not take into account thermal fluctuations. The formalism developed in Sec. II allows one to construct a model based on Eq. (66) which is consistent with statistical mechanics. Since relaxation towards equilibrium is governed by $\dot{s}=-$ const. $\times \sinh \left(s / s_{0}\right)$, the obvious choice for $F(s)$ is

$$
F(s)=f_{0} \cosh \left[\frac{s}{s_{0}}\right] .
$$

The Langevin equation corresponding to Eq. (68) is then

$$
\dot{s}=-\mu \frac{f_{0}}{s_{0}} \sinh \left[\frac{s}{s_{0}}\right]+\xi(t) .
$$

We now proceed to investigate this model, being particularly interested in to which extent it reproduces the predictions of Eq. (66).

First the nonlinear steady-state viscosity is considered. At low temperatures it is possible to derive an analytical expression for the viscosity. The derivation is given here for a general $F(s)$. If $\langle s\rangle$ denotes the average of $s$ during a steady shear flow, one has from Eq. (12) at low temperatures where fluctuations are small 


$$
\dot{\gamma} J(\langle s\rangle)=\mu \frac{d F}{d s}(\langle s\rangle) .
$$

The quantity $\langle s\rangle$ is a function of $\dot{\gamma}$. If the derivative of this function is denoted by $\langle s\rangle^{\prime}$, Eq. (70) implies by differentiation

$$
J+\dot{\gamma} \frac{d J}{d s}\langle s\rangle^{\prime}=\mu \frac{d^{2} F}{d s^{2}}\langle s\rangle^{\prime} .
$$

Combining this with Eq. (20) leads to

$$
J=\langle s\rangle^{\prime}\left[\mu \frac{d^{2} F}{d s^{2}}-\beta \dot{\gamma}\left(\frac{d F}{d s} J-\langle s\rangle\right]\right] .
$$

For $\beta \rightarrow \infty$ the term in the inner parentheses must vanish. In conjunction with Eq. (70) one finds

$$
\langle s\rangle=\frac{\mu}{\dot{\gamma}}\left[\frac{d F}{d s}(\langle s\rangle)\right]^{2},
$$

or for the viscosity

$$
\eta=\frac{1}{\mu}\left(\frac{\langle s\rangle}{\frac{d F}{d s}(\langle s\rangle)}\right)^{2}
$$

For the cosh model we thus have

$$
\eta=\eta_{0}\left(\frac{\langle s\rangle / s_{0}}{\sinh \left(\langle s\rangle / s_{0}\right)}\right)^{2},
$$

where

$$
\eta_{0}=\frac{1}{\mu} \frac{s_{0}^{4}}{f_{0}^{2}}
$$

is the linear viscosity. Perhaps surprisingly, one does not recover the Eyring viscosity expression

$$
\eta=\eta_{0} \frac{\langle s\rangle / s_{0}}{\sinh \left(\langle s\rangle / s_{0}\right)}
$$

but, as is clear from Fig. 2, the cosh model viscosity may be fitted reasonably well by Eq. (77).

Consider now relaxation towards equilibrium from a nonequilibrium state with stress $s$. At low temperatures $J(s)$ may be found explicitly from Eq. (21), which for $\beta \rightarrow \infty$ reduces to

$$
J(s)=\frac{s_{0}^{2}}{f_{0}} \frac{s / s_{0}}{\sinh \left(s / s_{0}\right)} .
$$

When substituted into Eq. (12) this leads to, in the zero noise limit,

$$
\dot{\gamma}=\frac{f_{0}}{s_{0}^{2}} \frac{\sinh \left(s / s_{0}\right)}{s / s_{0}} \dot{s}+\mu \frac{f_{0}^{2}}{s_{0}^{3}} \frac{\sinh ^{2}\left(s / s_{0}\right)}{s / s_{0}} .
$$

This looks nothing like Eq. (66). But again we find the nonlinear viscosity given by Eq. (75). Furthermore, for constant elongation, $s$ relaxes according to

$$
\dot{s}=-\mu \frac{f_{0}}{s_{0}} \sinh \left[\frac{s}{s_{0}}\right],
$$

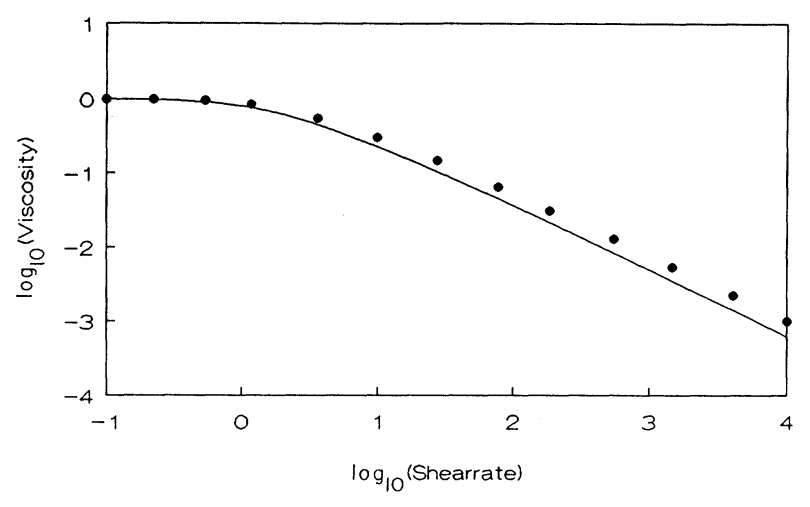

FIG. 2. Log-log plot of the steady-state nonlinear viscosity of the cosh model. The nonlinear viscosity is shown relative to the linear viscosity as a function of the dimensionless shear rate $\dot{\gamma} /\left(s_{0} / \eta_{0}\right)$. The nonlinear viscosity of the cosh model is not identical to the Eyring viscosity of Eq. (77) (marked by dots).

as expected from Eq. (69).

A final correspondence of the cosh model to Tobolsky's phenomenological model is the frequency-dependent viscosity. As is the case for any differential constitutive relation [1], Eq. (66) reduces to the Maxwell model in the linear limit. In the cosh model one might expect a more interesting frequency dependence, at least at high temperatures where the sinh factor of Eq. (69) cannot be replaced by a $s^{2}$ factor. But it turns out that even at high temperatures the autocorrelation function $\langle s(0) s(t)\rangle_{0}$ is actually very close to an exponential (which corresponds to the Maxwell model). What happens is that the logarithmic $s$ relaxation of Eq. (80), even at quite short times, is killed by the $s$ diffusion due to the noise term.

\section{Power-law model}

As a final example we briefly discuss the power-law model where

$$
F(s)=f_{0}\left|\frac{s}{s_{0}}\right|^{n} \quad(n>0) .
$$

The fact that $F(s)$ is nonanalytical at $s=0$ is insignificant. The case $n=2$ is the Gaussian model and the $n \rightarrow \infty$ limit is the box model.

The power-law model makes sense only for $n>\frac{3}{2}$. To prove this, the low-temperature limit is considered. For $\beta \rightarrow \infty, J(s)$ may be evaluated asymptotically from Eq. (21). Writing $s^{\prime n}-s^{n}=n s^{n-1}\left(s^{\prime}-s\right)$, one gets

$$
\begin{aligned}
J(s) & =\beta \int_{s}^{\infty} d s^{\prime} s^{\prime} e^{-\beta\left(f_{0} / s_{0}^{n}\right) n s^{n-1}\left(s^{\prime}-s\right)} \\
& \propto s\left(s^{n-1}\right)^{-1}=s^{2-n} .
\end{aligned}
$$

Thus, whenever $\dot{\gamma}$ is positive there is a "force" proportional to $s^{2-n}$ working to increase $s$. The "restoring force" from the potential varies as $s^{n-1}$. This force must dominate at large $s$ in order to avoid $s$ running off to infinity, thus $n-1>2-n$ or $n>\frac{3}{2}$. Mathematically, there is no normalizable stationary state whenever $n<\frac{3}{2}$. 
The border case $n=\frac{3}{2}$ leads to a model which is well defined up to a certain shear rate, above which $s$ runs off to infinity. This corresponds to there being a phase transition at a definite shear rate to a state of infinitely high viscosity.

To estimate the viscosity we use Eq. (73), which implies

$$
\dot{\gamma} \propto \frac{1}{s}\left(\frac{d F}{d s}\right)^{2} \propto s^{2 n-3} .
$$

This implies

$$
\eta=s / \dot{\gamma} \propto \dot{\gamma}^{(4-2 n) /(2 n-3)} .
$$

For $\frac{3}{2}<n<2$ the model exhibits shear thickening, whereas for $2<n$ there is shear thinning. The case $n=2$ gives a shear-rate-independent viscosity, as shown already.

\section{DISCUSSION}

The model of stress fluctuations considered in the present paper involves several nontrivial assumptions. The liquid is regarded as divided into "regions," and correlations between stress fluctuations of different regions are ignored completely. These assumptions are made for simplicity, but may be unrealistic since elastic forces are long ranged. The region picture becomes even harder to justify when a shear flow is considered. Such a flow deforms the regions and the picture can only be maintained whenever the longest correlation time is smaller than the inverse shear rate.

The main result of the paper is the proof that, if equilibrium stress fluctuations follow a Langevin equation, there is only one possible stress dynamics in nonequilibrium which is consistent with the principle of virtual work. Not only is the nonlinear response uniquely determined, but this is true also for the stress fluctuations in nonequilibrium. Crucial to this theorem is the assumption of a Langevin dynamics for the stress. This is a phenomenological postulate, but it should be emphasized that Langevin dynamics is the "canonical" guess if one is to discuss dynamics purely from a knowledge of equilibrium statistical mechanics. There is no other way of estimating the dynamics from a knowledge of only the equilibrium free energy $F(s)$. But, of course, this does not guarantee that the Langevin equation leads to correct results.

The assumption of linear coupling of the shear rate "field" in Eq. (12) is not essential. In fact, it is easy to show that the principle of virtual work implies the coupling must be linear. This is because the shear rate does not occur in Eq. (16).

The formalism developed may be generalized by replacing the term "stress" (which is the transverse momentum current) by any other current. One thus has a method for predicting the nonlinear response from a knowledge of equilibrium current fluctuations. The considering of currents as independent degrees of freedom has become popular in recent years, being the basis of extended irreversible thermodynamics $[12,13]$. The approach of Sec. II may be regarded as a statistical- mechanical counterpart to extended irreversible thermodynamics.

To illustrate the formalism a few simple models were studied in Sec. III. The Gaussian model leads to an exactly linear response even at large shear rates, reducing simply to the standard Maxwell model. The fact that the Gaussian model is linear is quite satisfactory, since a similar result is valid in ordinary statistical mechanics. Here, strictly Gaussian equilibrium fluctuations of, e.g., the magnetization, implies a field-independent magnetic susceptibility.

A more interesting model is the box model. It predicts a nonlinear viscosity (because there is a maximum possible stress), and a spectrum of relaxation times. In equilibrium this spectrum is not really visible, however; the autocorrelation function $\langle s(0) s(t)\rangle_{0}$ is approximately an exponential, leading to almost a Maxwell-type frequency dependence of the viscosity. Only in the relaxation from a strongly nonequilibrium state does the spectrum play any significant role, and even here the lowest eigenfrequency dominates the overall picture.

The cosh model was constructed to mimic Tobolsky's phenomenological model for stress relaxation. But although the zero noise relaxation equation is equal to Tobolsky's [Eq. (66)], the predictions of the model are not identical to those of Eq. (66). This is an illustration of a point made by van Kampen [7] that, by adding a noise term to a phenomenological model of the form $\dot{s}=f(s)$, some of the properties of the equation are lost because of the noise. In the case of the cosh model, the properties are retained in a qualitative sense, though. Thus, there is an Eyring-like viscosity in the model (Fig. 2), and the frequency dependence of the phenomenological model and the model of Eq. (69) are almost equal. The latter point may seem surprising, given the fact that the cosh model leads to a logarithmic relaxation towards zero in the zero noise limit (which, as is well known, corresponds to a spectrum of relaxation times varying like $\tau^{-1}$ ). However, just as in the box model, the spectrum is not significant in equilibrium where the noise term completely dominates the autocorrelation function, resulting in an almost exponential decay.

The cosh model corresponds to an exponentially increasing free energy $F(s)$. The case of $F(s)$ increasing following a power law was also considered in Sec. III. [The case of a logarithmically increasing $F(s)$ leads to an inconsistent model where $s$ runs off to infinity whenever $\dot{\gamma} \neq 0$.] The power-law model is consistent only for $n>\frac{3}{2}$, $n=\frac{3}{2}$ being a border case where the model makes sense for not too large shear rates. Whenever $\frac{3}{2}<n<2$ the model exhibits shear thickening, whereas $2<n$ corresponds to the experimentally more common case of shear thinning. The case $n=2$ is the Gaussian model, and the limit $n \rightarrow \infty$ is the box model. A closer analysis than given in Sec. III reveals that the shear thickening in the case $\frac{3}{2}<n<2$ is a consequence of one not having $J(s) \rightarrow 0$ as $|s| \rightarrow \infty$ [while for $2<n J(s) \rightarrow 0$ as $|s| \rightarrow \infty$ ]. Since $J(s)$ may be interpreted as an $s$-dependent infinite frequency shear modulus, the study of the power-law model leads to a novel view on the origin of nonlinearity: Nonlinearity may be viewed as a consequence of a stress- 
dependent infinite frequency shear modulus $G_{\infty}$. The case when $G_{\infty}$ increases with increasing stress corresponds to shear thickening while a decreasing $G_{\infty}$ (i.e., softening) corresponds to shear thinning. The Gaussian linear case $n=2$ corresponds to a stress independent $G_{\infty}$. A final thing to be noted about the power-law model is that in the zero noise limit this model has a power-law time dependence of the stress relaxation, as is easy to show.

The predictions of the models are approximately on the level of differential constitutive relations: these have realistic nonlinear steady-state viscosities but only a simple Maxwell frequency dependence of the viscosity [1]. Compared to differential constitutive relations, the presently considered models have the advantage of being consistent with statistical mechanics. Thus, the method presented may be regarded as a means of modifying differential constitutive relations to include thermal fluctuations. The modification, however, is nontrivial in the sense that the differential constitutive relation is not recovered exactly in the low-temperature limit, as shown in detail for the cosh model.

Several important features of polymeric liquids are not mimicked by the models of Sec. III. The predicted almost single relaxation-time frequency dependence of the viscosity is very far from that observed in polymeric liquids. Another important point which is not captured by the models is the fact that experimentally, nonlinearity sets in at a shear rate about equal to the frequency marking the onset of frequency dependence of the viscosity
$[1,14]$. Finally, the temperature dependence of the viscosity is weak and there is a well-defined viscosity in the zero-temperature limit. While this last point could be handled by assuming the mobility $\mu$ is temperature dependent, the two other points are quite serious, indeed. A further objection is the fact that in the present model there is time reversibility in a steady shear flow: The steady-flow Langevin equation obeys detailed balance for a suitably chosen energy function [compare Eq. (26)]. In a real flow one expects a genuine violation of time reversibility.

In conclusion, the types of models studied in Sec. III are not satisfactory as models of reality. To arrive at more realistic models one has to consider several stress coordinates interacting with each other, for instance by taking into account the spatial variation of the stress. This leads to a field theory in which the free energy is a functional of the stress field. If this function has several minima, the Langevin dynamics gives thermally activated relaxation times (just as in the description of chemical reactions), and thereby more realistic temperature and frequency dependences. Also, it may be shown that in a model with more than one degree of freedom there is genuine time irreversibility in any shear flow.

\section{ACKNOWLEDGMENT}

This work was supported by the Danish Natural Science Research Council.
[1] R. B. Bird, R. C. Armstrong, and O. Hassager, Dynamics of Polymeric Liquids, 2nd ed. (Wiley, New York, 1987), Vols. 1 and 2.

[2] M. Doi and S. F. Edwards, The Theory of Polymer Dynamics (Clarendon, Oxford, 1986).

[3] A. Tobolsky and H. Eyring, J. Chem. Phys. 11, 125 (1942).

[4] A. V. Tobolsky and R. D. Andrews, J. Chem. Phys. 13, 3 (1945).

[5] R. D. Andrews, N. Hofman-Bang, and A. V. Tobolsky, J. Polym. Sci. 3, 669 (1948).

[6] R. Becker, Theory of Heat (Springer, Berlin, 1967).

[7] N. G. van Kampen, Stochastic Processes in Physics and Chemistry (North-Holland, Amsterdam, 1981).
[8] M. Doi, J. Chem. Phys. 79, 5080 (1983).

[9] J. D. Ferry, Viscoelastic Properties of Polymers, 3rd ed. (Wiley, New York, 1981).

[10] P. M. Morse and H. Feshback, Methods of Theoretical Physics (McGraw-Hill, New York, 1953).

[11] M. R. Speigel, Mathematical Handbook, Schaum's Outline Series in Mathematics (McGraw-Hill, New York, 1968).

[12] D. Jou, J. Casas-Vázquez, and G. Lebon, Rep. Prog. Phys. 51, 1105 (1988).

[13] D. Jou, J. Casas-Vázquez, and G. Lebon, J. Non-Equilib. Thermodyn. 17, 383 (1992).

[14] W. W. Graessley, Adv. Polym. Sci. 16, 1 (1974). 